\title{
Therapeutic results in elderly patients with prostate cancer: chronological comparison in a single community hospital
}

\author{
Takehiko Okamura1 , Hidetoshi Akita ${ }^{1}, K^{1}$ enji Yamada1, Daichi Kobayashi', Yasuhiko Hirose ${ }^{1}$, \\ Takahiro Kobayashi ${ }^{1}$, Yutaro Tanaka ${ }^{1}$, Taku Naiki ${ }^{2}$ and Takahiro Yasui ${ }^{2}$ \\ ${ }^{1}$ Department of Urology, Anjo Kosei Hospital, Japan \\ ${ }^{2}$ Department of Nephro-Urology, Nagoya City University Graduate School of Medical Sciences, Japan
}

\begin{abstract}
Objective: There are few reports of the long-term outcomes of elderly patients with prostate cancer. We analyzed data from our institution from the past 12 years, including the patient history, treatment methods, and prognosis of patients with prostate cancer aged 80 years or more.

Patients and Methods: A total of 179 cases of prostate cancer in patients aged 80 years or more were retrospectively evaluated. We divided them chronologically into groups A, B, C, and D: Group A included 40 cases from 2002-2004; Group B, 48 cases from 20052007; Group C, 46 cases from 2008-2010; and Group D, 45 cases from 2011-2013.

Results: Sixty-one (30\%) patients changed treatment course. Interestingly, no cancer deaths occurred in the patients who changed treatment course. Although 14 (7.8\%) cancer deaths occurred (A: $\mathrm{B}: \mathrm{C}: \mathrm{D}=4: 4: 6: 0$, respectively), all occurred in 2011 or later.

Conclusion: In our study, over 50 patients who underwent treatment survived for 5 years or more. By treating prostate cancer in elderly patients when appropriate, we can lower the mortality rate due to prostate cancer. Our results support the active treatment of prostate cancer in elderly patients.
\end{abstract}

Key words: prostate cancer, hormonal therapy, elderly patients

(J Rural Med 2016; 11(2): 59-62)

Received: August 17, 2016

Accepted: October 19, 2016

Correspondence: Takehiko Okamura, Department of Urology, J. A. Aichi Anjo Kosei Hospital, 28 Higashihirokute, Anjo-cho, Anjo 4468602, Japan

E-mail: hiko2546@kosei.anjo.aichi.jp

This is an open-access article distributed under the terms of the Creative Commons Attribution Non-Commercial No Derivatives (by-nc-nd) License $<$ http://creativecommons.org/licenses/by-nc-nd/4.0/>.

\section{Introduction}

Over the last decade, remarkable advancements have been made in treating prostate cancer, including robot-assisted laparoscopic prostatectomy (RALP) ${ }^{1,2)}$, new radiotherapy techniques such as brachytherapy, intensity-modulated radiation therapy (IMRT), proton therapy ${ }^{3-5)}$, taxane-based chemotherapy, and hormonal therapy with abiraterone or enzalutamide ${ }^{6}$. However, in Japan, conventional androgen deprivation therapy (hormone therapy) continues to be the primary initial treatment option for the majority of patients, especially the elderly ${ }^{7}$.

Moreover, prostate-specific antigen (PSA) screening has been increasing annually, with a corresponding increase in the sensitivity of PSA testing kits. In Europe and the United States, on the other hand, standard treatment guidelines do not recommend PSA screening for men over 80 years of age $^{8,9)}$. Little has been reported on the long-term outcomes of elderly patients with prostatic carcinoma ${ }^{10,11}$. We analyzed data from our institution from the past 12 years, including patient history, treatment methods, and prognosis in prostate cancer patients aged 80 and older.

\section{Patients and Methods}

A total of 179 cases of prostate cancer diagnosed between 2002 and 2013 in patients aged 80 years or more were retrospectively evaluated. The median age was 83.0 (range, $80-96$ ) years. PSA values were 1.5 to $2695.9 \mathrm{ng} / \mathrm{ml}$ (mean $167.9, \mathrm{SD} \pm 584.7 \mathrm{ng} / \mathrm{ml})$. In 64 cases, PSA values were less than 10; in 82 cases, values were between 10 and 100; in 33 cases, they were greater than 100; and in two cases, no data were available. According to T-stage classification, the number of T1, T2, T3, and T4 tumors were 36, 52, 45, and 41 , respectively. Five cases could not be staged.

Chronologically, four historical groups were compared: 


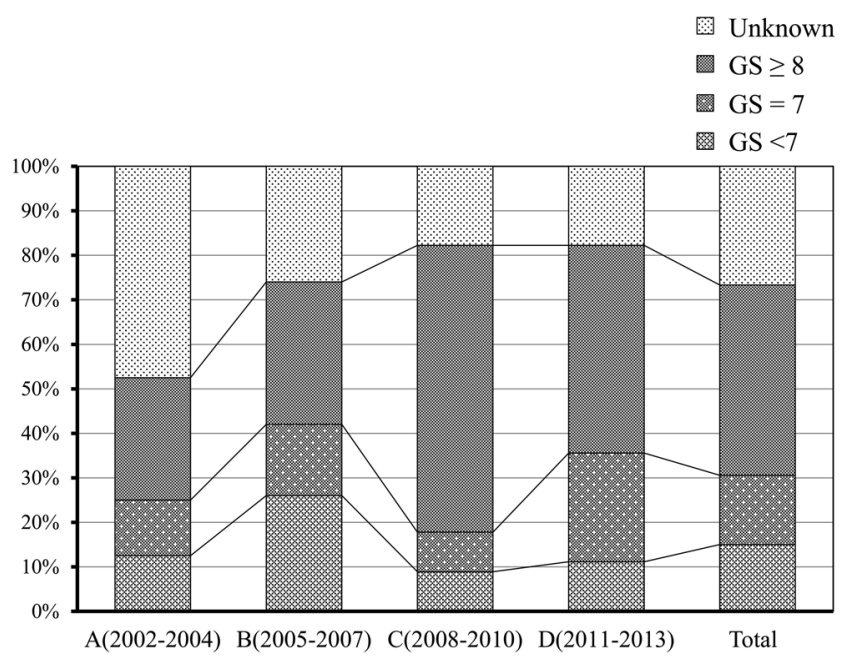

Figure 1 Percentage of Gleason scores (GS) by chronological groups.

Group A included 40 cases from 2002-2004; Group B, 48 cases from 2005-2007; Group C, 46 cases from 2008-2010; and Group D, 45 cases from 2011-2013. Complications, such as heart failure, diabetes mellitus, or hypertension, were reported in 74 cases, while none were reported in the remaining 107 cases.

All patients provided informed consent, and this study protocol has been approved by our hospital's committee on human research.

Statistical analysis was performed using Fisher's exact test. Cumulative (overall and cancer specific) survival rates were estimated using the Kaplan-Meier method, and the significance of differences between curves was tested by the log-rank test. A value of $p<0.05$ was considered statistically significant. All statistical analyses were performed using SPSS Version 17.

\section{Results}

The follow-up period ranged from 1 to 136 months (mean 41.7, $\mathrm{SD} \pm 33.0$ months). Fifty-two patients were observed for more than 60 months. The percentage of patients with a Gleason score (GS) greater than 7 was significantly higher in 2008 and later (Figure 1, p = 0.0151). There were no significant differences in PSA values or T stages among the four groups. Initial treatments of these patients were as follows: 144 patients received maximum androgen blockage (MAB), 23 patients received a luteinizing hormone releasing hormone (LH-RH) analogue or anti-androgen monotherapy, 9 patients were put on watchful waiting, and 5 patients received MAB plus oral chemotherapy. Watchful waiting was introduced as a treatment option in 2005, but its frequency

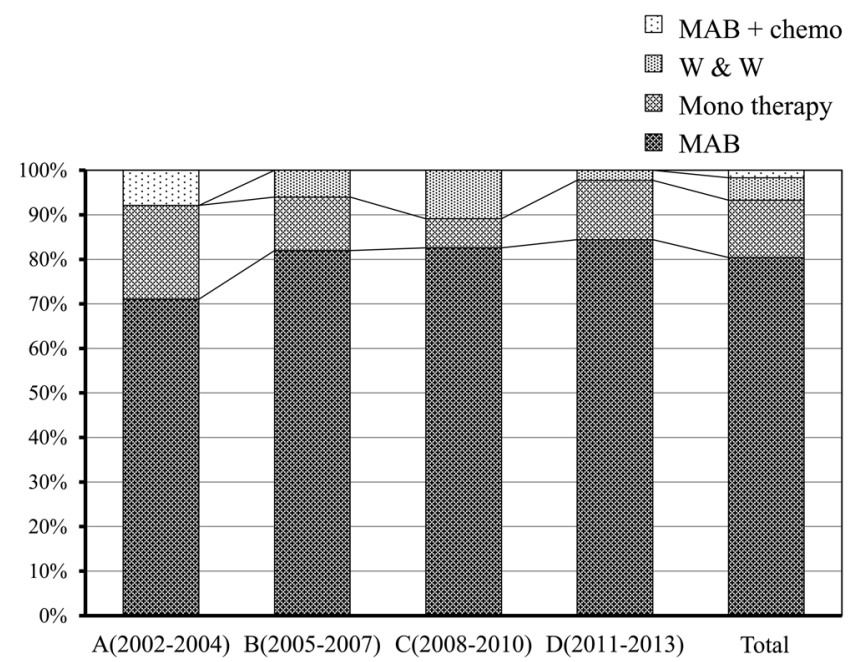

Figure 2 Treatments by chronological groups. MAB: maximum androgen blockage, W \& W: watchful waiting, Monotherapy: luteinizing hormone releasing hormone agonist monotherapy, Chemo: chemotherapy.

did not increase depending on the time period (Figure 2). Out of all of the cases, a total of $62(30 \%)$ patients changed treatment course, including anti-androgen turnover; interestingly, no cancer deaths occurred in these cases. In 8 patients, treatment changed twice; in 6 patients, three times; and in 5 patients, treatment changed four times. Of these 62 patients, 60 patients changed treatment due to initial treatment failure, but there was no significant difference in the number of treatment changes among the four groups.

Although $14(7.8 \%)$ cancer deaths occurred in total (in groups A: B: C: D were 4: 4: 6: 0, respectively), all occurred in 2011 or later (Figure 3).

No significant differences were found in overall survival by time period (Figure 4).

\section{Discussion}

Our data have demonstrated the potential for treatment efficacy in elderly prostate cancer patients. Out of 179 patients, more than 50 survived for 5 years or more after undergoing treatment. By treating prostate cancer in elderly patients with androgen deprivation therapy when appropriate, we can reduce mortality 70,11 .

In our hospital, the frequency of prostatic carcinoma has been increasing each year. However, in patients aged 80 years and more, that rate has not increased in over 10 years.

The incidence of prostate cancer has been increasing rapidly thanks to longer lifespans and Western dietary habits $^{12,13}$. According to the American Urological Association 
Deaths from other causes

图 Deaths from cancer

Alive

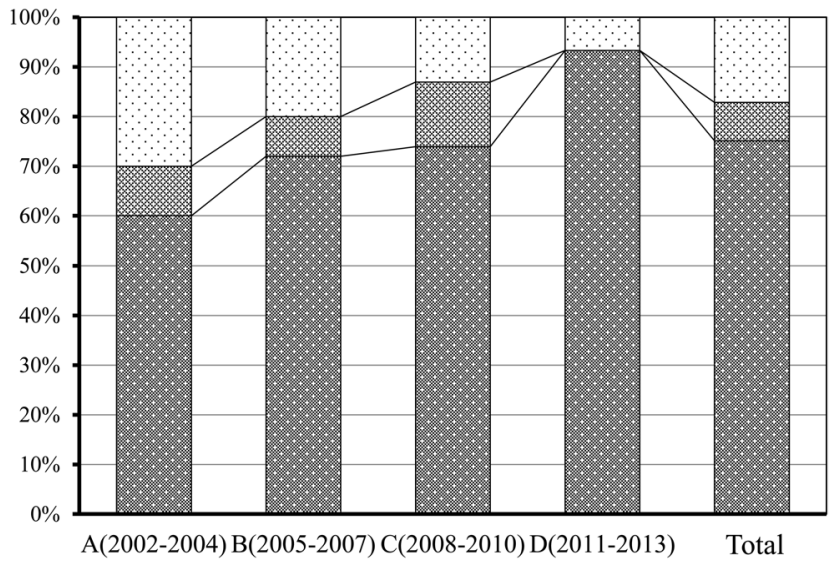

Figure 3 Mortality by chronological groups.

(AUA) guidelines for the early detection of prostate cancer, routine PSA screening is not recommended for patients 70 years of age and older (especially for those above age 74), because of the lack of evidence showing any benefit ${ }^{8}$. Additionally, PSA screening is not recommended for patients with a life expectancy of less than 10 to 15 years (Recommendation; Evidence Strength Grade C). These guidelines are in place to avoid overdiagnosis and overtreatment of those with low-risk disease, since the harms from overtreatment outweigh any potential benefits and may negatively impact quality of life ${ }^{8}$. Meanwhile guidelines from the European Association of Urology (EAU) indicate that an individualized risk-adapted strategy for early detection might be appropriate for certain men with good performance status and a life expectancy of at least $10-15$ years".

However, ethical debates in this regard persist, and some reports have described a high rate of malignancy among elderly patients with prostate cancer in Asian countries ${ }^{10-12}$. Elderly men with localized prostate cancer come from a variety of backgrounds, and age should be just one of several factors to consider when determining optimal treatment ${ }^{13,14}$. Our data suggest that many elderly men with prostate cancer may benefit from androgen deprivation therapy and survive longer than previously expected. Moreover, we experienced no cancer death in patients who changed treatments. Conventional androgen deprivation therapy and further aggressive treatment following anti-androgen therapies were thought to be quite effective in our patients. In addition, many new types of hormonal drugs are now available ${ }^{6}$, and these drugs are expected to improve prognosis. By treating prostate cancer in selected elderly patients when appropriate, our data suggest that we can lower the mortality rate

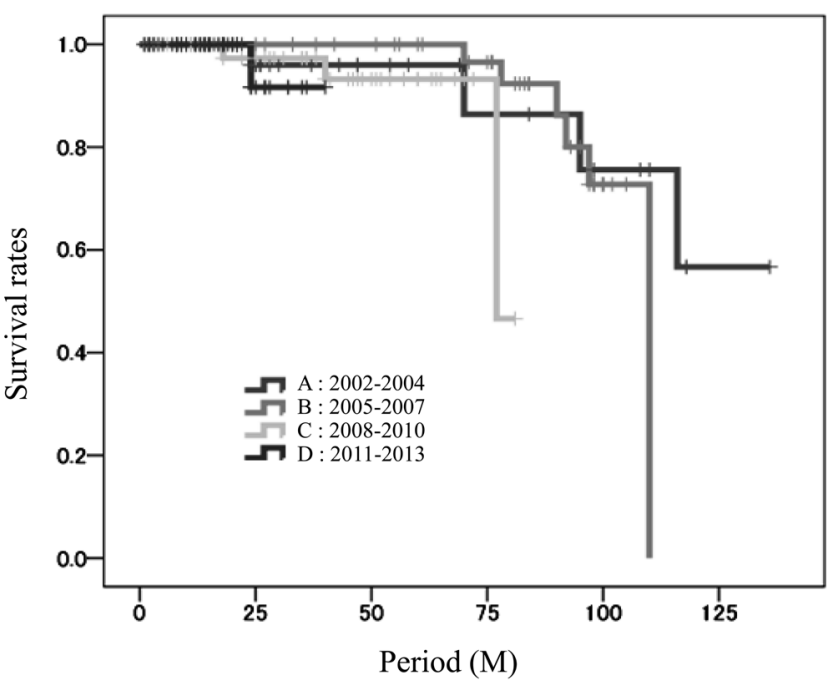

Figure 4 Kaplan-Meier analysis of overall survival.

from prostate cancer by providing multiple treatment options.

Limitations of our study include the absence of an estimation of the quality of life, the lack of a control group to compare a cancer-free population with prostate cancer patients, and the relatively small sample size. Further large cohort studies could clarify which patients would benefit the most from androgen deprivation therapy in the future.

In conclusion, in our study, over 50 patients survived for 5 years or more by undergoing treatment. By treating prostate cancer in elderly patients with the use of androgen deprivation therapy when appropriate, we can lower the mortality rate from this disease. Our results support the active treatment of prostate cancer in elderly patients. Further investigation and large-scale analysis in other Japanese hospitals are needed.

Conflict of Interest: None declared.

\section{References}

1. Ploussard G, de la Taille A, Moulin M, et al. Comparisons of the perioperative, functional, and oncologic outcomes after robot-assisted versus pure extraperitoneal laparoscopic radical prostatectomy. Eur Urol 2014; 65: 610-619. [Medline] [CrossRef]

2. Ficarra V, Novara G, Ahlering TE, et al. Systematic review and meta-analysis of studies reporting potency rates after robot-assisted radical prostatectomy. Eur Urol 2012; 62: 418430. [Medline] [CrossRef]

3. Evans JR, Zhao S, Daignault S, et al. PROSTQA Study Consortium Patient-reported quality of life after stereotactic body radiotherapy (SBRT), intensity modulated radiotherapy 
(IMRT), and brachytherapy. Radiother Oncol 2015; 116: 179184. [Medline] [CrossRef]

4. Bell LJ, Cox J, Eade T, et al. Determining optimal planning target volume and image guidance policy for post-prostatectomy intensity modulated radiotherapy. Radiat Oncol 2015; 10: 151. [Medline] [CrossRef]

5. Hoppe BS, Bryant C, Sandler HM. Radiation for prostate cancer: intensity modulated radiation therapy versus proton beam. J Urol 2015; 193: 1089-1091. [Medline] [CrossRef]

6. Gillessen S, Omlin A, Attard G, et al. Management of patients with advanced prostate cancer: recommendations of the St Gallen Advanced Prostate Cancer Consensus Conference (APCCC) 2015. Ann Oncol 2015; 26: 1589-1604. [Medline] [CrossRef]

7. Fujimoto H, Nakanishi H, Miki T, et al. Oncological outcomes of the prostate cancer patients registered in 2004: report from the Cancer Registration Committee of the JUA. Int J Urol 2011; 18: 876-881. [Medline] [CrossRef]

8. AUA guideline (2013) https://www.auanet.org/education/ guidelines/prostate-cancer-detection.cfm.

9. EAU guideline (2014) http://uroweb.org/wp-content/
uploads/1607-Prostate Cancer.

10. Pierorazio PM, Humphreys E, Walsh PC, et al. Radical prostatectomy in older men: survival outcomes in septuagenarians and octogenarians. BJU Int 2010; 106: 791-795. [Medline] [CrossRef]

11. Kunz I, Musch M, Roggenbuck U, et al. Tumour characteristics, oncological and functional outcomes in patients aged $\geq 70$ years undergoing radical prostatectomy. BJU Int 2013; 111(3 Pt B): E24-E29. [Medline] [CrossRef]

12. Alvarez-Cubero MJ, Pascual-Geler M, Rivas A, et al. Lifestyle and dietary factors in relation to prostate cancer risk. Int J Food Sci Nutr 2015; 66: 805-810. [Medline] [CrossRef]

13. Sonoda T, Nagata Y, Mori M, et al. A case-control study of diet and prostate cancer in Japan: possible protective effect of traditional Japanese diet. Cancer Sci 2004; 95: 238-242. [Medline] [CrossRef]

14. Mitsuzuka K, Koie T, Narita S, et al. Changes in indications and oncological outcomes of radical prostatectomy after 2000 data from 1268 Japanese patients treated with radical prostatectomy between 2000 and 2009. Jpn J Clin Oncol 2013; 43: 821-826. [Medline] [CrossRef] 